\title{
Diffraction limited near-infrared imaging of the Red Rectangle by bispectral analysis
}

\author{
P. Cruzalèbes ${ }^{1}$, E. Tessier ${ }^{2}$, B. Lopez ${ }^{3}$, A. Eckart ${ }^{4}$ and D. Tiphène ${ }^{5}$ \\ 1 Observatoire de la Côte d'Azur, Département Fresnel (URA CNRS 1361), Av. Copernic, F-06130 Grasse, France \\ 2 Laboratoire d'Astrophysique (URA CNRS 708), Observatoire de Grenoble, Université Joseph-Fourier, BP. 53X, \\ F-38041 Grenoble Cedex, France \\ 3 Observatoire de la Côte d'Azur, Département Fresnel (URA CNRS 1361), BP. 229, F-06034 Nice Cedex 4, France \\ 4 Max-Planck-Institut für Extraterrestrische Physik, Postfach 1603, D-85740 Garching, Germany \\ 5 Observatoire de Paris-Meudon, Département Spatial, URA CNRS, 92195 Meudon Cedex, France
}

Received June 23; accepted September, 1995

\begin{abstract}
We present a new method for infrared speckle imaging mainly based on the bispectral analysis algorithm applied to extended objects. The efficiency of the reconstruction procedure is increased by a relaxation process making use of redundancies in the pupil plane. The method is applied to near- and mid-infrared data sets of the Red Rectangle (CRL 915) in $K, L^{\prime}$ and $M$-bands leading to diffraction limited maps of the post-AGB (Asymptotic Giant Branch) bipolar nebula. Studying the morphology of the Red Rectangle at sub-second of arc scale allows to decompose the near and mid-infrared broadband spectrum in two parts: the radiated light from the central part of the nebula and the radiated light from the lobes to the north and south. This decomposition indicates that, in addition to scattering of the light from a central source, thermal extended emission from warm dust may also be important at arsecond and subarcsecond scales in this bipolar nebula.
\end{abstract}

Key words: infrared: ISM: continuum — planetary nebulae: individual (CRL 915) — stars: individual (HD44179) — techniques: image processing — techniques: interferometric

\section{Introduction}

Large bidimensional infrared array detectors have been available since only recently. Their advent is causing important changes in infrared astronomy (Gatley 1987). There are two ways to reach the optical/IR diffraction limit of a large ground-based telescope: using the statistical properties of the atmosphere in post-processing shortexposure speckled frames (Labeyrie 1970); or on-line correcting the atmosphere disturbed wavefront by adaptive optics (Babcock 1953).

In the field of speckle imaging, a big step has been taken thanks to the introduction of third-order correlation. Bispectral analysis is a seeing deconvolution method processing in the Fourier plane, based on triple correlations (Weigelt 1977). Although it has been first successfully applied to objects consisting of point-like sources (stellar clusters) as HD 97950 AB in NGC 3603 (Hofmann \& Weigelt 1986), or Eta Carinae (Ebersberger \& Weigelt 1986), not many applications to extended ( $\sim 1$ to a few arcseconds) objects have been published.

Send offprint requests to: P. Cruzalèbes (cruzalebes@obsazur.fr)
We present an adaptation of the bispectral analysis method to such objects. Our bispectrum phase relaxation method has a similar effect as a least-square fitting method (Glindemann et al. 1992) but has the advantage of being free from input models. Let us mention that phase relaxation algorithms have also been developed for KnoxThompson analysis (Hardy et al. 1977; Drummond et al. 1988). We successfully applied this method to analyze near-infrared observations of the Red Rectangle. This object is a post-AGB source with a bipolar nebula appearance known in the visible. The nickname of Red Rectangle refers to the remarkable rectangular symmetry of the visible image in the red light (Cohen et al. 1975). The scattering of the stellar radiation by the circumstellar dust is assumed to be responsible for the shape of the nebula. The central object (HD 44179) is a ninth $V$-magnitude source of spectral type B9-A0 III. It is possibly a binary star (Cohen et al. 1975).

The geometry of the circumstellar envelope is not fully understood. Yusef-Zadeh \& Morris (1984) have shown, using some radiative transfer simulations, that a disk-like structure for the envelope reproduces the visible image of the Red Rectangle. 
The diffraction limited images we present here in the near- and mid-infrared ( $K, L^{\prime}$ and $M$ bands) with an angular resolution better than 0.2 arcsec give a new insight of the nearby environment of the mass loosing late type star HD 44179. The extension of the associated nebula still exists up to $\lambda=5 \mu \mathrm{m}$ and has a rectangular shape (as first order).

A comparison of the reconstructed images among each other as well as a comparison to published $K$-band Shiftand-add and Knox-Thompson reconstructions (Eckart \& Duhoux 1990) clearly reveal some complex structural features. Let us mention that the reduction here described was done in part using the IRSI software of the previous reference. These structures may be related to heterogeneousnesses (clumps) in the dusty outflow. The presence of the supposed companion, although not excluded, is not obvious in our analysis.

\section{Speckle observations at the CFHT}

Observations were carried out at CFHT (Canada-FranceHawaii Telescope) with the INSU-OPM (Institut National des Sciences de l'Univers, Observatoire de Paris-Meudon) infrared camera CIRCUS (Lacombe et al. 1989) on 1990 December 3. Since 1989, this camera allows speckle-type acquisition in the $K, L^{\prime}$ and $M$ bands (Tessier et al. 1994). Due to the large readout noise of the chip $\left(2500 \mathrm{e}^{-}\right)$ used then and its small size $(32 \times 32)$, observations were restricted to the $L^{\prime}(3.87 \mu \mathrm{m})$ and $M(4.8 \mu \mathrm{m})$ bands (Tessier 1993). More than 5000 frames of $0.16 \mathrm{~s}$ integration time were obtained at $L^{\prime}$ and $M$ on the Red Rectangle with an image scale of 0.103 arcsec per pixel and a field of view of 3.3 arcsec. The seeing was between 0.5 arcsec and 1 arcsecond FWHM in the visible (airmass was smaller than 1.3). The speckle transfer function was estimated on a nearby reference star (HR 2267). We switched every few minutes between the source and its reference in order to sample the temporal seeing variations (condition of stationarity) so as to minimize seeing effects during the reduction process (Perrier 1988). We estimated the contribution of the detector and of the background by integrating images of the sky close to the source $\left(40^{\prime \prime}\right.$ away) during the same amount of time. The integration time was selected to achieve the best angular resolution with a sufficient signal-to-noise (Tessier 1993), the limiting parameter being the temporal coherence of the turbulent atmosphere.

\section{Speckle observations at the KPNO}

Another set of speckle data comes from the IR speckle camera (Beckers et al. 1988) obtained at the Kitt Peak National Observatory 3.8-m (Mayall) telescope. Used at $K(2.2 \mu \mathrm{m})$ band, the camera provides an image scale of $0.058^{\prime \prime}$ per pixel, the size of each frame being 64 by 64 pixels.
Observations were carried out in 1987 November and consist of 360 specklegrams of the Red Rectangle and 240 specklegrams of the point source (IRC-10118) of around $1 / 30 \mathrm{~s}$ integration time. At $0.5 \mu \mathrm{m}$ the seeing during those nights was typically $1.0^{\prime \prime}$, that corresponds to $0.75^{\prime \prime}$ at $2.2 \mu \mathrm{m}$, as compared to a speckle size of $0.12^{\prime \prime}$.

\section{Data reduction process}

The data reduction process is based on the bispectral analysis algorithm and consists of the following image processing steps (Fig. 1):

- Calculation of the ensemble averaged power spectrum and bispectrum of the speckle interferograms (source, reference and sky).

- Noise correction of the sky background (in the power spectrum and the bispectrum, for the source and for the reference).

- Calibration of the object power spectrum and bispectrum by compensation of the speckle interferometry transfer function in the power spectrum and bispectrum with the reference spectral and bispectral transfer functions.

- Determination of the phase of the object Fourier transform from the bispectrum.

- Determination of a high-resolution image of the object from the object Fourier transform.

- Cleaning of the reconstructed map with the point spread function.

Important steps of this process are examined in the following sections. We assume that the raw data sets have been already pre-processed frame by frame, by applying the dead pixel corrections, zero level offsets (subtraction of the background), flat fielding and selection of the "good" frames (in terms of seeing parameters).

\subsection{The bispectral analysis method}

The bispectral analysis method leads to diffraction-limited images in spite of image degradation by the atmosphere and by telescope aberrations. Short exposures of the Red Rectangle and of its reference produce speckle patterns in the image plane of the telescope.

Each two-dimensional instantaneous frame $I(\alpha)$ can be written as the convolution product (denoted by $\otimes$ ) of the object $O(\alpha)$ with the combined telescope and atmospheric instantaneous point spread function $S(\alpha)$

$$
I(\alpha)=O(\alpha) \otimes S(\alpha) .
$$

Since the Fourier transform of a convolution is an ordinary product, the Fourier transform of Eq. (1) can be written as

$$
\hat{I}(\nu)=\hat{O}(\nu) \times \hat{S}(\nu)
$$




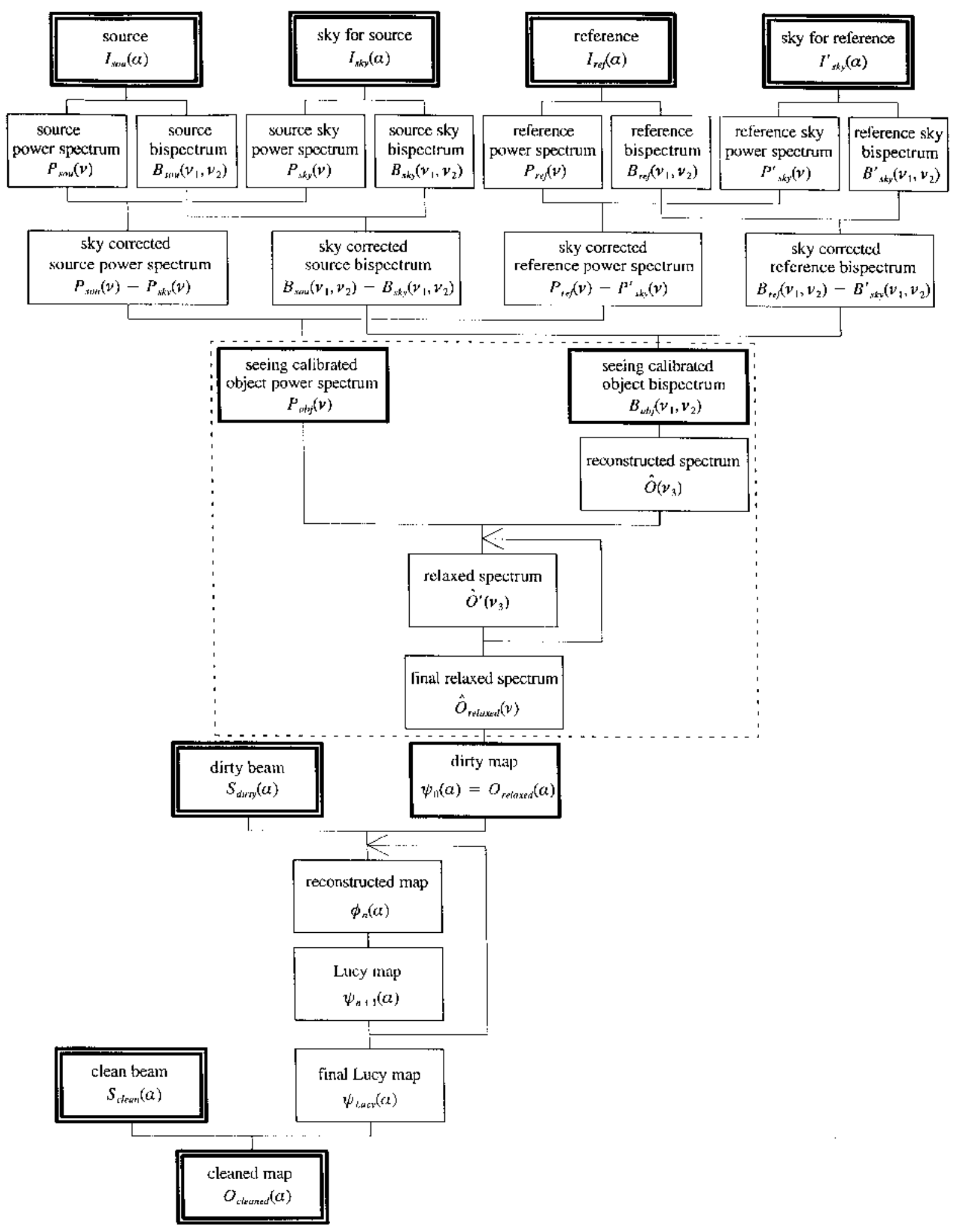

Fig. 1. a) Flow chart of the speckle bispectral analysis algorithm. The process can be divided in 5 successive steps: calculation of averaged power spectra and bispectra (source, reference and skies), sky correction (unbiased power spectra and bispectra), seeing calibration with the reference, iterating complex Fourier spectrum recovery and relaxation of the spectral phase, and finally cleaning with the Lucy iterating algorithm. Note that the core of the algorithm is the relaxation procedure (marked with a dashed line) 


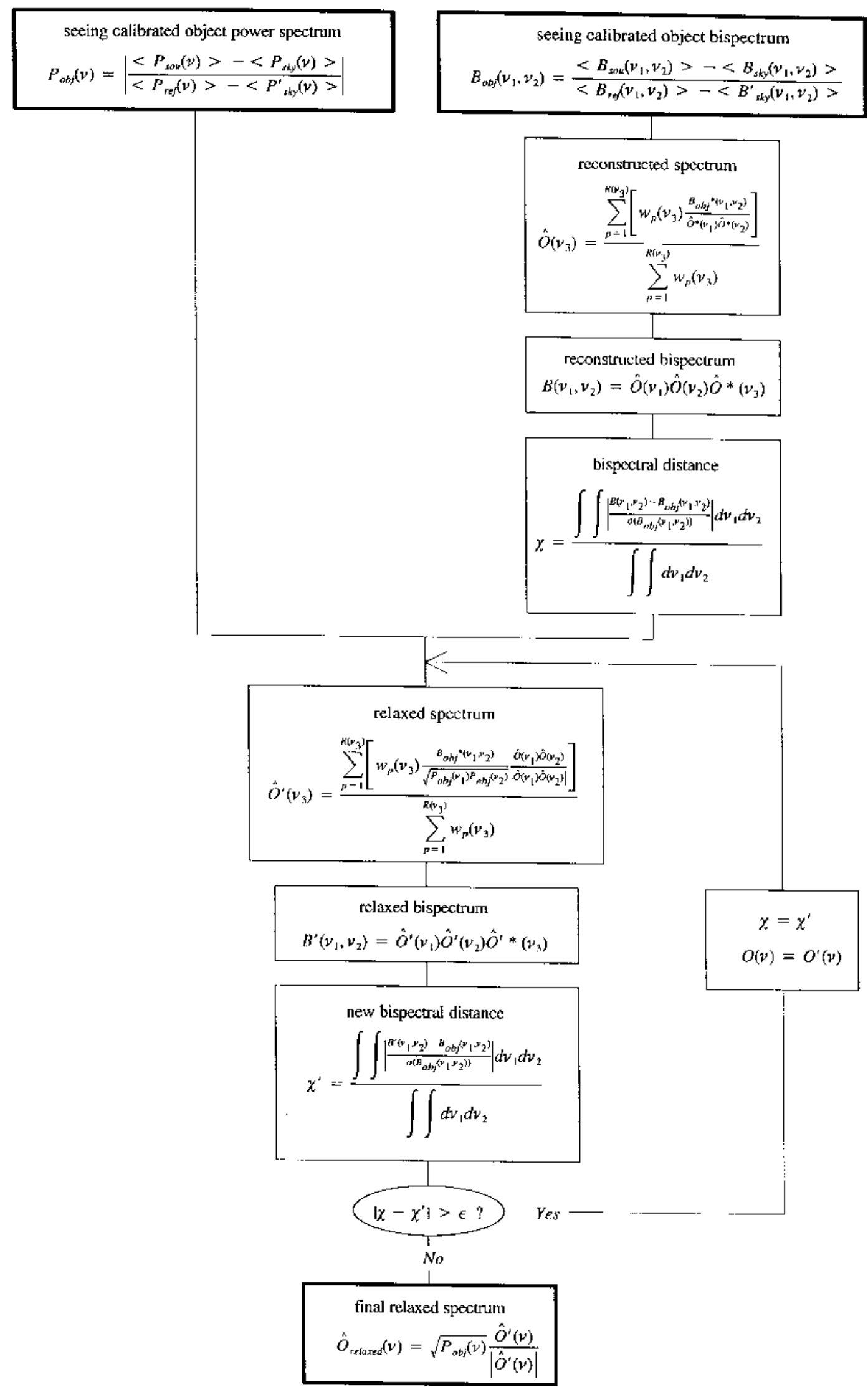

Fig. 1. b) Detailed flow chart of the relaxation procedure (central part of Fig. 1a) indicating each operation involved and its result. For more explanation report to Sects. 4.6 and 4.7 
where $\hat{S}(\nu)$ describes the instantaneous transfer function at the spatial frequency $\nu$, affected by the atmospheric turbulence.

Quantitative information is retrieved from a statistical analysis of a sequence of these instantaneous speckle frames.

The triple correlation procedure, also called speckle masking or bispectral analysis (Weigelt 1977; Lohmann et al. 1983), is a natural generalization of the Labeyrie's selfcorrelation or power spectrum analysis (Labeyrie 1970). It consists in computing the averaged triple correlation or, which is equivalent, its Fourier transform, called the energy bispectrum of the image.

The classical Labeyrie's analysis consists in calculating the ensemble averaged power spectrum given by

$$
<|\hat{I}(\nu)|^{2}>=|\hat{O}(\nu)|^{2}<|\hat{S}(\nu)|^{2}>.
$$

It is the product of the energy spectrum of the object with the speckle transfer function which can be calibrated on a point source (unresolved star) as long as the process is stationary both in time and space (Roddier 1988).

To reconstruct a general object $O(\alpha)$ from speckle interferometric measurements, we need both the modulus and the phase of its Fourier transform $\hat{O}(\nu)$. Power spectrum analysis is well suited to estimate the object energy power spectrum $|\hat{O}(\nu)|^{2}$, i.e. the modulus of its Fourier transform, but the main drawback of this data reduction process is the loss of phase information.

To preserve some object phase information we use the image bispectrum given by

$$
\begin{aligned}
<\hat{I}\left(\nu_{1}\right) \hat{I}\left(\nu_{2}\right) \hat{I}^{*}\left(\nu_{1}+\nu_{2}\right)> & =\hat{O}\left(\nu_{1}\right) \hat{O}\left(\nu_{2}\right) \hat{O}^{*}\left(\nu_{1}+\nu_{2}\right) \\
& <\hat{S}\left(\nu_{1}\right) \hat{S}\left(\nu_{2}\right) \hat{S}^{*}\left(\nu_{1}+\nu_{2}\right)>
\end{aligned}
$$

It is the product of the bispectrum of the object with the bispectral transfer function. As for the speckle transfer function, the bispectral transfer function can also be calibrated on a point source (Roddier 1988).

\subsection{Linearization of the bispectrum}

The manipulation of the bispectrum, which is a 4dimensional complex function, presents some difficulties, mainly because the required memory space (central and virtual memory) is enormous when reducing large images. The number of bispectral elements increases as the fourth power of the array size $N$ (von der Lühe \& Pehlemann 1988). The full bispectrum has $\frac{9 N^{4}}{16}$ index combinations if sums of indexes are kept within the principal spectrum range. Exploiting all possible symmetry relations allows to reduce the number of elements of the bispectrum to

$\frac{3 N}{8}\left(\frac{N}{4}+1\right)\left(\frac{N^{2}}{2}+N+1\right)=\frac{3 N^{4}}{64}+\frac{9 N^{3}}{32}+\frac{15 N^{2}}{32}+\frac{3 N}{8}$.
A bispectrum reduced to a non-symmetric subset would still consist of about $5.910^{4}$ complex elements when $N=32$, or about $8.610^{5}$ elements when $N=64$.

Consequently, we would rather represent the bispectrum in a one-dimensional form because of memory allocation problems. This has the supplementary advantage of sequential processing: for loading as well as for integration of the bispectrum, this data order minimizes paging. Classification of bispectral elements takes into account the way the object spectrum will be recovered. It is based on classification of the Fourier vectors $\nu_{1}$ and $\nu_{2}$, starting from the origin of the Fourier plane and proceeding in a circular spiral out to the diffraction limit. Thus, the bivectors $\left(\nu_{1}, \nu_{2}\right)$ are classified according to the following rules: $\left|\nu_{1}\right|,\left|\nu_{2}\right|,\left|\nu_{1}+\nu_{2}\right| \leq\left|\nu_{\mathrm{c}}\right| ;\left|\nu_{1}\right| \leq\left|\nu_{2}\right| ;$ and $\left|\nu_{1}\right| \leq\left|\nu_{0}\right| ;$ where $\left|\nu_{c}\right|=\frac{D}{\lambda}$ is the diffraction limit frequency of the telescope, $D$ being its diameter, and $\nu_{0}$ is an adjustable cutoff frequency, whose modulus is given by the signalto-noise ratio in the power spectrum. Choosing small $\left|\nu_{0}\right|$ forces $\hat{I}\left(\nu_{1}\right)$ to have a high signal-to-noise ratio and also reduces the number of bispectral elements. In the bispectrum 4-dimensional space, this corresponds to nearaxis points. Increasing $\left|\nu_{0}\right|$ introduces a larger number of redundant triple products with low signal-to-noise ratio (SNR). Although they contribute to an overall gain in SNR if properly weighted, they dramatically increase the computational efforts.

An optimum of SNR versus computational effort can be reached by choosing a cutoff in $\left|\nu_{0}\right|$ out to which triple products with sufficiently large SNR are used by the reconstruction process. A typical value for $\left|\nu_{0}\right|$ is the long exposure cutoff frequency $\frac{r_{0}}{\lambda}$, where $r_{0}$ is the Fried parameter (Fried 1979; Roddier 1981).

Let us notice that distinct $\left(\nu_{1}, \nu_{2}\right)$ bivectors can produce the same $\nu_{3}=\nu_{1}+\nu_{2}$ resulting vector. The number of such pairs is called the redundancy of the pupil at the frequency $\nu_{3}$ (Cruzalèbes et al. 1992). High degree of redundancy is found around the center of the Fourier plane (small frequencies), while high frequencies have a small degree of redundancy. The redundancy function has to be circular-symmetric around the center of the Fourier plane. This property can be used to check the completeness (and/or quality) of the one-dimensional arrangement of triple products used for the reconstruction of the complex source spectrum.

\subsection{Noise correction of the bispectrum}

Although the average zero level offset due to the sky background has been removed to each frame in the preprocessing step, some residual contribution of the sky background to the noise may still exist. To get rid of it, one has to subtract its effects on both the power spectrum and the bispectrum. As a first order approximation, its contribution can be corrected by subtracting the power spectrum and bispectrum of the sky with the power spectra 
and bispectra of the source and its calibrator (the reference star). Theoretically, the sky is isotrope (i.e. no structure is present, thus the phase is null), but, as a matter of fact, the images recorded on the sky may show some structures mostly due to the detector (even after flat fielding).

Let $I_{\text {sou }}(\alpha)$ be a speckle frame of the source (Fig. 1), we can write that the sky corrected frame of the source is $I_{\text {sou }}(\alpha)-I_{\text {sky }}(\alpha)$.

If we neglect the cross terms that vanish in presence of zero averaging noise, the instantaneous sky corrected power spectrum of the source can be written as $P_{\text {sou }}(\nu)-$ $P_{\text {sky }}(\nu)$, difference between the raw power spectrum of the source and the power spectrum of the sky for the source (called sky corrected source power spectrum in Fig. 1a). Similarly, the instantaneous sky corrected bispectrum of the source becomes $B_{\text {sou }}\left(\nu_{1}, \nu_{2}\right)-B_{\text {sky }}\left(\nu_{1}, \nu_{2}\right)$ (called sky corrected source bispectrum in Fig. 1a).

For the reference, the noise correction follows the same rules. The sky corrected frame of the reference is $I_{\text {ref }}(\alpha)-$ $I_{\text {sky }}^{\prime}(\alpha)$, while the instantaneous sky corrected power spectrum of the reference (called sky corrected reference power spectrum in Fig. 1a) is $P_{\text {ref }}(\nu)-P_{\text {sky }}^{\prime}(\nu)$, difference between the raw power spectrum of the reference (the square of the MTF) and the power spectrum of the sky for the reference, while the instantaneous sky corrected bispectrum of the reference is $B_{\text {ref }}\left(\nu_{1}, \nu_{2}\right)-B_{\text {sky }}^{\prime}\left(\nu_{1}, \nu_{2}\right)$, (called sky corrected reference bispectrum in Fig. 1a).

\subsection{Seeing calibration of the bispectrum}

In their article, Lohmann et al. (1983) claimed that no calibration of the transfer function is required in order to recover the true object phase from the bispectra. We, nevertheless, performed the calibration of the bispectrum: if one assumes that the atmosphere does not have any structural component in phase, the optics, as well as the detector itself, may introduce some.

The bispectral transfer function that appears in Eq. (4) can, in principle, be calibrated on a reference star. Its amplitude takes high values when either $\nu_{1}, \nu_{2}$ or $\nu_{1}+\nu_{2}$ is smaller than the seeing cutoff frequency $\frac{r_{0}}{\lambda}$. Then it drops off very rapidly up to the limit where $\nu_{1}, \nu_{2}$ and $\nu_{1}+\nu_{2}$ approach the diffraction cutoff frequency $\frac{D}{\lambda}$. Its amplitude is seeing-dependent and varies with the inverse square of the number of speckles in the image for spatial frequencies from $\frac{r_{0}}{\lambda}$ to $\frac{D}{\lambda}$ (Roddier 1988). Its phase is seeing-independent and equal to zero. It is insensitive to telescope aberrations as long as these are small compared to the effects of turbulence.

Using Eq. (4), the sky corrected and seeing calibrated object bispectrum (called object bispectrum in Fig. 1) is given by

$$
B_{\text {obj }}\left(\nu_{1}, \nu_{2}\right)=\frac{<B_{\text {sou }}\left(\nu_{1}, \nu_{2}\right)>-<B_{\text {sky }}\left(\nu_{1}, \nu_{2}\right)>}{<B_{\text {ref }}\left(\nu_{1}, \nu_{2}\right)>-<B_{\text {sky }}^{\prime}\left(\nu_{1}, \nu_{2}\right)>}
$$

Let us notice that, if one could measure the sky background simultaneously to the source and if a reference star is available in the isoplanatic patch, it should be much more preferable to sky correct and seeing calibrate instantaneously the bispectrum as follows

$$
B_{\mathrm{obj}}\left(\nu_{1}, \nu_{2}\right)=<\frac{\hat{I}_{\mathrm{ss}}\left(\nu_{1}\right) \hat{I}_{\mathrm{sS}}\left(\nu_{2}\right) \hat{I}_{\mathrm{sS}}^{*}\left(\nu_{1}+\nu_{2}\right)}{\hat{I}_{\mathrm{ss}}^{\prime}\left(\nu_{1}\right) \hat{I}_{\mathrm{ss}}^{\prime}\left(\nu_{2}\right) \hat{I}_{\mathrm{ss}}^{\prime}\left(\nu_{1}+\nu_{2}\right)}>,
$$

where $\hat{I}_{\text {ss }}=\hat{I}_{\text {sou }}(\nu)-\hat{I}_{\text {sky }}(\nu)$ and $\hat{I}_{\mathrm{ss}}^{\prime}(\nu)=\hat{I}_{\text {ref }}(\nu)-\hat{I}_{\text {sky }}^{\prime}(\nu)$ are the Fourier transforms of the instantaneous sky corrected speckle frames of the source and of the reference respectively. This instantaneous calibration leads to better results because the reference star and the skies have been taken in exactly the same conditions of turbulence as the source. Unfortunately, this is not the case with the Red Rectangle data.

In order to avoid zero divisions when calibrating the bispectrum with Eqs. (6) or (7), we have implemented an iterating algorithm, based on Van Cittert (1931). The output estimate of this algorithm converges to the solution of the normal division after an infinite number of iterations.

The principle of the method is the following. Let be $R=\frac{P}{Q}$. If $|Q| \ll 1$ the calculation of $R$ may rapidly diverge. One estimate of $R$ could be:

$$
R_{n}=P \times \sum_{i=0}^{n}(1-Q)^{i} .
$$

According to the fact that $\sum_{i=0}^{\infty}(1-Q)^{i}=\frac{1}{Q}$, it is easy to demonstrate that $\lim _{n \rightarrow \infty} R_{n}=R$.

In the case where the modulus of the denominator of the second term in Eqs. (6) or (7) becomes smaller than $10^{-1}$, we typically used about ten iterations to estimate the ratio. In the other case, we calculated the ratio by normal division.

\subsection{Tracing the errors}

The statistical errors associated with the source bispectrum, the reference bispectrum, the sky bispectrum for the source and the sky bispectrum for the reference are estimated from the standard deviations of the bispectral distributions, $\sigma\left(B_{\text {sou }}\right), \sigma\left(B_{\text {ref }}\right), \sigma\left(B_{\text {sky }}\right)$ and $\sigma\left(B_{\text {sky }}^{\prime}\right)$ respectively. From Eq. (6), the relative error of the object bispectrum is given by

$$
\begin{aligned}
\frac{\sigma\left(B_{\text {obj }}\left(\nu_{1}, \nu_{2}\right)\right)}{\left|B_{\text {obj }}\left(\nu_{1}, \nu_{2}\right)\right|} & =\frac{\sqrt{\sigma^{2}\left(B_{\text {sou }}\left(\nu_{1}, \nu_{2}\right)\right)+\sigma^{2}\left(B_{\text {sky }}\left(\nu_{1}, \nu_{2}\right)\right)}}{\left|B_{\text {sou }}\left(\nu_{1}, \nu_{2}\right)-B_{\text {sky }}\left(\nu_{1}, \nu_{2}\right)\right|} \\
& +\frac{\sqrt{\sigma^{2}\left(B_{\text {ref }}\left(\nu_{1}, \nu_{2}\right)\right)+\sigma^{2}\left(B_{\text {sky }}^{\prime}\left(\nu_{1}, \nu_{2}\right)\right)}}{\left|B_{\text {ref }}\left(\nu_{1}, \nu_{2}\right)-B_{\text {sky }}^{\prime}\left(\nu_{1}, \nu_{2}\right)\right|}
\end{aligned}
$$




\subsection{Reconstruction of the object Fourier spectrum}

In order to reconstruct the object spectrum from the calibrated object bispectrum, we used a recursive scheme (Bartelt et al. 1984; Weitzel et al. 1992) starting at the origin of the Fourier plane

$$
\hat{O}^{*}\left(\nu_{3}\right)=\frac{B_{\mathrm{obj}}\left(\nu_{1}, \nu_{2}\right)}{\hat{O}\left(\nu_{1}\right) \hat{O}\left(\nu_{2}\right)} .
$$

The two object spectral elements $\hat{O}\left(\nu_{1}\right)$ and $\hat{O}\left(\nu_{2}\right)$ must be known to deduce the third element $\hat{O}\left(\nu_{3}=\right.$ $\nu_{1}+\nu_{2}$ ) (called reconstructed spectrum in Fig. 1) from the calibrated object bispectrum $B_{\mathrm{obj}}\left(\nu_{1}, \nu_{2}\right)$. The initial conditions, to start the recursive process, set the phase at the origin of the Fourier space to zero and the object spectrum to

$$
\hat{O}(0)=\sqrt[3]{B_{\mathrm{obj}}(0,0)}
$$

The phases of the two first adjacent object spectral elements (remember that the spectral vectors are classified according to a spiral beginning at the origin) are also set to zero. This can be done without penalty, because these spatial frequencies are small and because an arbitrary choice of the central phases only affects the absolute position of the reconstructed image (Lohmann et al. 1983). Alternatively these phase values can be taken from the long exposure image.

Since redundancies (number of different $\left(\nu_{1}, \nu_{2}\right)$ bivectors producing the same sum vector; see Cruzalèbes et al. 1992) have been taken into account in the formation of the bispectrum (see Sect. 4.2), each point of the object spectrum $\hat{O}\left(\nu_{3}\right)$ receives many estimations that are averaged using the signal to noise ratio (inverse of the relative error) as weighting factors. Let us designate by $R\left(\nu_{3}\right)$ the number of redundancies for the spectral vector $\nu_{3}=\nu_{1}+\nu_{2}$. Each estimate of $\hat{O}\left(\nu_{3}\right)$ is numbered by the $p$ index, varying from 1 to $R\left(\nu_{3}\right)$. Equation (10) becomes

$$
\hat{O}_{p}\left(\nu_{3}\right)=\frac{B_{\mathrm{obj}}{ }^{*}\left(\nu_{1}, \nu_{2}\right)}{\hat{O}^{*}\left(\nu_{1}\right) \hat{O}^{*}\left(\nu_{2}\right)} .
$$

Thus, the weighted estimate of $\hat{O}\left(\nu_{3}\right)$ is

$$
\hat{O}\left(\nu_{3}\right)=\frac{\sum_{p=1}^{R\left(\nu_{3}\right)} \omega_{p}\left(\nu_{3}\right) \times \hat{O}_{p}\left(\nu_{3}\right)}{\sum_{p=1}^{R\left(\nu_{3}\right)} \omega_{p}\left(\nu_{3}\right)},
$$

where the weighting factor $\omega_{p}\left(\nu_{3}\right)$ is given by the signal to noise ratio

$$
\begin{aligned}
\omega_{p}\left(\nu_{3}\right) & =\frac{\left|\hat{O}_{p}\left(\nu_{3}\right)\right|}{\sigma\left(\hat{O}_{p}\left(\nu_{3}\right)\right)} \\
& =\left(\frac{\sigma\left(B_{\mathrm{obj}}\left(\nu_{1}, \nu_{2}\right)\right)}{\left|B_{\mathrm{obj}}\left(\nu_{1}, \nu_{2}\right)\right|}+\frac{\sigma\left(\hat{O}\left(\nu_{1}\right)\right)}{\left|\hat{O}\left(\nu_{1}\right)\right|}+\frac{\sigma\left(\hat{O}\left(\nu_{2}\right)\right)}{\left|\hat{O}\left(\nu_{2}\right)\right|}\right)^{-1},
\end{aligned}
$$

which is the inverse of the relative error deduced from Eq. (12).

Assuming normal statistics, the statistical error of the weighted estimate of $\hat{O}\left(\nu_{3}\right)$ is given by

$$
\sigma^{2}\left(\hat{O}\left(\nu_{3}\right)\right)=\frac{\sum_{p=1}^{R\left(\nu_{3}\right)} \omega_{p}^{2}\left(\nu_{3}\right) \times \sigma^{2}\left(\hat{O}_{p}\left(\nu_{3}\right)\right)}{\sum_{p=1}^{R\left(\nu_{3}\right)} \omega_{p}^{2}\left(\nu_{3}\right)} .
$$

Let us notice that there is not an unique way for the recursion process in bispectral analysis. Lohmann et al. (1983) present two of them. In general one want to use triple products with the highest Signal-to-Noise Ratio up to the highest possible spatial frequency. This by itself implies that the reconstruction scheme is source dependent. As a consequence of that, a relaxation method has been developed to smooth irregularities due to the propagation of the noise in the recursive procedure of reconstruction. The method is based on the concept that the phase of the reconstructed spectrum can be relaxed if the modulus is introduced in the procedure as a constraint.

\subsection{Relaxation of the triple phase factors}

Since the reconstruction of the object complex spectrum has been achieved by averaging the noisy bispectrum, the recovered object spectrum is noise affected. As a result of that, the reconstructed object bispectrum, which can be calculated with the recovered object spectrum, does not agree perfectly with the input calibrated object bispectrum, obtained from Eq. (6). An iterative spectral relaxation procedure is performed, initially developed for Knox-Thompson analysis (Hardy et al. 1977; Drummond et al. 1988), that smooths irregularities and converges to a solution for the object reconstructed complex spectrum compatible both with the input calibrated bispectrum and with the input calibrated power spectrum.

The principle of the method is to introduce in the reconstruction procedure of Eq. (12) the calibrated power spectrum as a constraint for the modulus of the spectral elements $\hat{O}\left(\nu_{1}\right)$ and $\hat{O}\left(\nu_{2}\right)$ as following:

$$
\hat{O}_{p}\left(\nu_{3}\right)=\frac{B_{\mathrm{obj}}{ }^{*}\left(\nu_{1}, \nu_{2}\right)}{\sqrt{P_{\mathrm{obj}}\left(\nu_{1}\right) P_{\mathrm{obj}}\left(\nu_{2}\right)}} \frac{\hat{O}\left(\nu_{1}\right) \hat{O}\left(\nu_{2}\right)}{\left|\hat{O}\left(\nu_{1}\right) \hat{O}\left(\nu_{2}\right)\right|} .
$$

Thus, only the phase is kept as a result for the reconstruction from the calibrated bispectrum, while the modulus is constrained by the calibrated power spectrum. Figure $1 \mathrm{~b}$ shows the reconstruction and relaxation procedures, which are the core of the general algorithm presented in Fig. 1a.

$P_{\text {obj }}(\nu)=\left|\frac{\left\langle P_{\text {sou }}(\nu)>-<P_{\text {sky }}(\nu)\right\rangle}{\left\langle P_{\text {ref }}(\nu)>-<P_{\text {sky }}^{\prime}(\nu)\right\rangle}\right|$ is the sky corrected and seeing calibrated object power spectrum obtained from 
Eq. (3) with the original power spectra of source, reference, sky for the source and sky for the reference. Let us notice that, in order to avoid zero divisions when calibrating the power spectrum, we have also used the iterating Van Cittert algorithm described in Eq. (8) but applied to the calibration of the power spectrum.

In each loop of the recursive relaxation, the new relaxed spectrum is calculated from the input seeing calibrated bispectrum and the old relaxed spectrum (Fig. 1b).

To check on convergence of the relaxation algorithm, the distance between the input calibrated object bispectrum and the relaxed object bispectrum (triple product of the relaxed spectrum) is calculated (Fig. 1b) as:

$$
\chi=\frac{\iint\left|\frac{B\left(\nu_{1}, \nu_{2}\right)-B_{\text {obj }}\left(\nu_{1}, \nu_{2}\right)}{\sigma\left(B_{\text {obj }}\left(\nu_{1}, \nu_{2}\right)\right)}\right| \mathrm{d} \nu_{1} \mathrm{~d} \nu_{2}}{\iint \mathrm{d} \nu_{1} \mathrm{~d} \nu_{2}}
$$

Convergence is reached when the bispectral distance defined in the above equation tends to a constant (and not null) value. On average, changes of the bispectrum then are of the order or smaller than the corresponding errors.

As a summary, one can say that the relaxation algorithm iteratively calculates the object complex spectrum making simultaneous use of the constraints imposed by the seeing and noise calibrated power spectrum and bispectrum.

\subsection{Image reconstruction}

After the last relaxation loop, one can extract the final reconstructed phase factor of the last reconstructed complex spectrum. When combined with the square root of the power spectrum, it gives an estimate of the final reconstructed object complex spectrum, being compatible both with the calibrated power spectrum and bispectral elements. The reconstructed map (called dirty map in Fig. 1) is obtained by inverse Fourier transformation of the final reconstructed complex spectrum (called final relaxed spectrum in Figs. 1a and 1b).

\subsection{Cleaning of the reconstructed maps}

Because the calibrated power spectrum has been calculated over a finite support in the Fourier space up to the diffraction limit, the reconstructed map clearly shows rings around quasi point like objects. To get rid of this effect, we "CLEANed" the reconstructed image of the object after relaxation (the dirty map in Fig. 1) with the inverse Fourier transform of the apodization filter we used in Fourier space (the dirty beam in Fig. 1) to have the power spectrum smoothly roll off at or close to the diffraction limit of the telescope. After a few 1000 iterations we reconvolved the final map with a Gaussian function (the clean beam in Fig. 1) with a full width at half maximum (FWHM) given by the diameter of the central lobe of the inverse Fourier transform of the apodization filter. As a cleaning algorithm we used the Lucy algorithm (Lucy 1974). This cleaning procedure makes the mapping algorithm as well as the quality and resolution in the final map (the cleaned map) much less dependent on the exact choice of the apodization filter.

The Lucy algorithm is an iterating nonlinear deconvolution method based on the comparison of the untreated input map $\psi_{0}(\alpha)$, also called the dirty map, with the current estimate of the deconvolved map $\psi_{n}(\alpha)$ convolved (denoted by $\otimes$ ) with the dirty beam $S_{\text {dirty }}(\alpha)$ (the experimental point-spread function). The comparison is performed by calculating a low-pass filtered ratio of the reconvolved estimate $\phi_{n}(\alpha)$ (called reconstructed map in Fig. 1 ) and the input map. This quotient is then used to calculate a new estimate $\psi_{n+1}(\alpha)$ (called Lucy map in Fig. 1 ) of the object $O(\alpha)$. The iterative process stops when the last estimate reconvolved with the dirty beam agrees within the measurement uncertainties with the untreated input map. The reconstructed map is the convolution of the final estimate (called final Lucy map in Fig. 1) with the clean beam $S_{\text {clean }}(\alpha)$.

The process is summarized by the following expressions:

- initialization of the process:

$$
\psi_{0}(\alpha)=O_{\text {relaxed }}(\alpha)
$$

- for $n=1$ to $N$ :

$$
\begin{gathered}
\phi_{n}(\alpha)=\psi_{n}(\alpha) \otimes S_{\text {dirty }}(\alpha) \\
\psi_{n+1}(\alpha)=\psi_{n}(\alpha) \times\left[\frac{O_{\text {relaxed }}(\alpha)}{\phi_{n}(\alpha)} \otimes S_{\text {dirty }}(\alpha)\right] \\
-\quad \\
O_{\text {cleaned }}(\alpha)=\psi_{N+1}(\alpha) \otimes S_{\text {clean }}(\alpha) .
\end{gathered}
$$

Due to the calculation of the quotient $\frac{O_{\text {relaxed }}(\alpha)}{\phi_{n}(\alpha)}$ in Eq. (20), the algorithm is sensitive to noise. Therefore, a weighting function has been implemented which is unity if the dirty map is higher than a given threshold and zero elsewhere. $\left[\frac{O_{\text {relaxed }}(\alpha)}{\phi_{n}(\alpha)} \otimes S_{\text {dirty }}(\alpha)\right]$ is only calculated in that area. To check on convergence of the algorithm, the root mean square between the input dirty map and the current Lucy deconvolved map reconvolved with the dirty beam is calculated.

The result of the Lucy deconvolution exhibits the following properties (Lucy 1974):

1. The reconstructed map exhibits a maximum degree of smoothness and continuity. 
2. The reconstructed map differs from the dirty map only by amounts that can ascribed to errors in the measurement.

3. The method is flux conserving.

4. After a infinite number of iterations the method converges to the maximum likelihood solution.

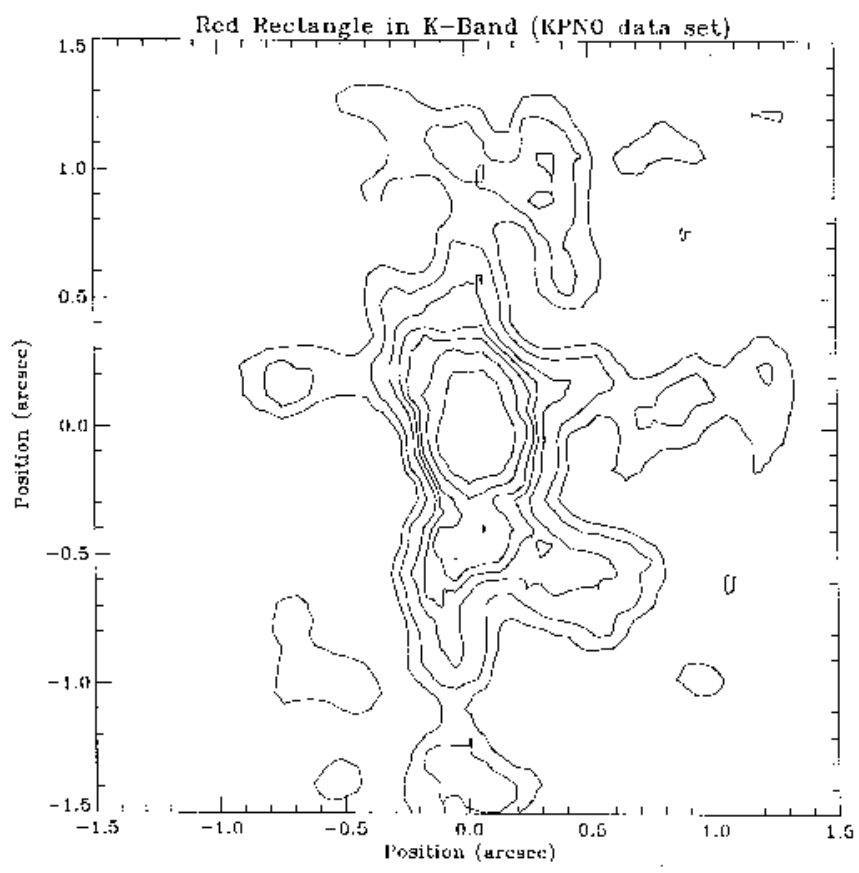

Fig. 2. Bilinear interpolated reconstructed map of the Red Rectangle in $K$-band. North is up and east is left. Field of view has been restricted to $3^{\prime \prime} \times 3^{\prime \prime}$. Final resolution is $0.2^{\prime \prime}$. Flux has been normalized to its maximum. Overplotted contours are $0.5,1,2,3,5,10$ and $20 \%$ of the maximum of intensity. Lowest contour is three times the standard deviation of the noise, measured in regions external to the nebula

Recent comparisons of different deconvolution algorithms applied both to point-like sources and to extended objects have shown that results of the nonlinear Lucy deconvolution are in very good agreement with those of the linear deconvolution obtained by division of Fourier transforms (Cunningham \& Anthony 1993).

Several thousand iterations were used for the Red Rectangle deconvolution. The resulting maps were then reconvolved with Gaussian functions of $0.2^{\prime \prime}$ in $K$-band, $0.3^{\prime \prime}$ in $L^{\prime}$-band and $M$-band FWHM. Although the theoretical diffraction limits are about $0.12^{\prime \prime}$ at $K$ for a 3.81 m telescope, $0.22^{\prime \prime}$ at $L^{\prime}$ and $0.28^{\prime \prime}$ at $M$ for a $3.58 \mathrm{~m}$ telescope, we have degraded a little the resolution of the reconstructed maps, in order to keep only the most significant features in the Red Rectangle.

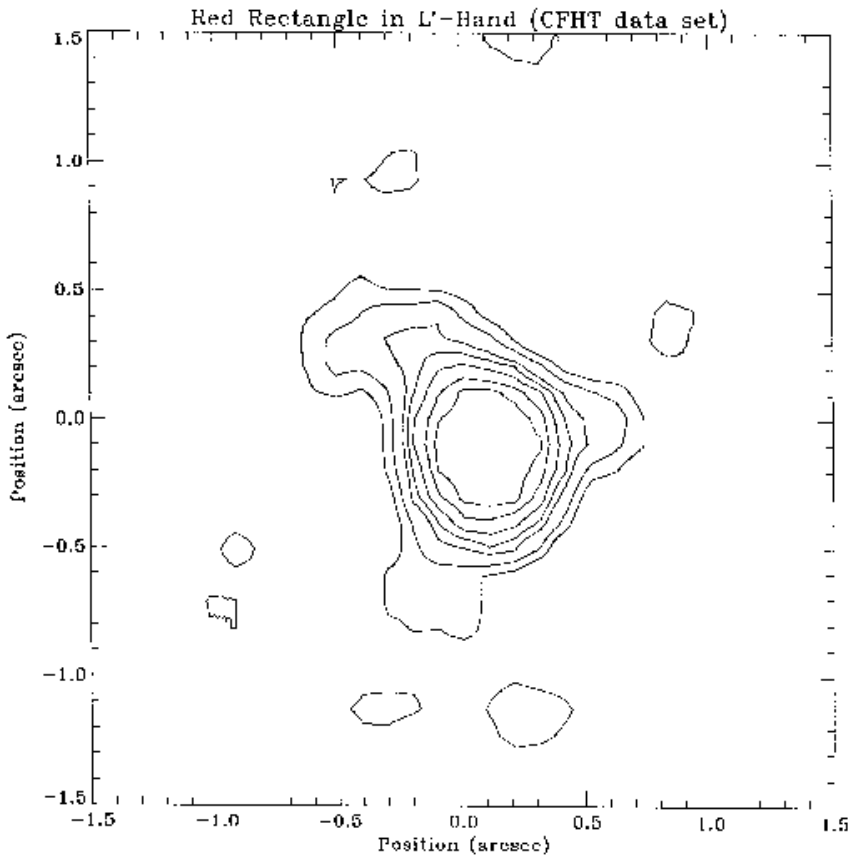

Fig. 3. Bilinear interpolated reconstructed map of the Red Rectangle in $L^{\prime}$-band. North is up and east is left. Field of view has been restricted to $3^{\prime \prime} \times 3^{\prime \prime}$. Final resolution is $0.3^{\prime \prime}$. Flux has been normalized to its maximum. Overplotted contours are $0.5,1,2,3,5,10$, and $20 \%$ of the maximum of intensity. Lowest contour is three times the standard deviation of the noise, measured in regions external to the nebula

\section{Discussion}

\subsection{Red Rectangle morphology}

The result of the bispectral analysis processing of about 360 short exposures of the Red Rectangle in the $K$ band is displayed in Fig. 2. The angular resolution after image reconstruction is of $0.15^{\prime \prime}$. The $L^{\prime}$ and $M$ band speckle reconstructions at a respective resolution of $0.2^{\prime \prime}$ and $0.3^{\prime \prime}$ are shown in Figs. 3 and 4. In each map the lowest contour level is about three times the standard deviation of the noise (measured in regions external to the nebula). The bispectral analysis processing in $L$ and $M$ bands was performed on about 5000 short exposure frames.

The general features of the nebula are the same in each of the near-IR images: the overall shape of the nebula is bipolar. Figure 5 illustrates schematically the geometry of the nebula mainly based on the $M$-band reconstructed map (Fig. 4) which shows the most extended structures (compared to the $K$-band and the $L^{\prime}$-band). The central part $0.7^{\prime \prime}$ in size lies between two side lobes, $1.2^{\prime \prime}$ in size for the North-eastern lobe, and $1^{\prime \prime}$ for the South-eastern one. The opening angle of the outflow indicated by the NIR extensions is of the order of $20^{\circ}$ (from North to East).

In $K$-band (Fig. 2) the result of our mapping is in excellent agreement with earlier reconstruction of the same 


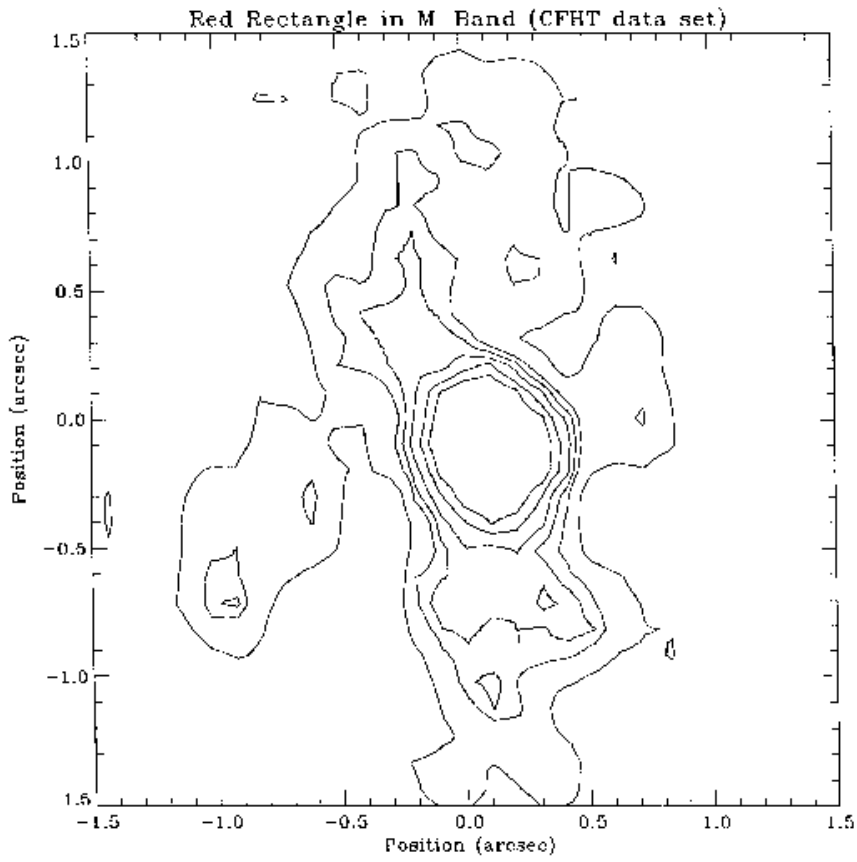

Fig. 4. Bilinear interpolated reconstructed map of the Red Rectangle in M-band. North is up and east is left. Field of view has been restricted to $3^{\prime \prime} \times 3^{\prime \prime}$. Final resolution is $0.3^{\prime \prime}$. Flux has been normalized to its maximum. Overplotted contours are $1,2,3,4,5,10$, and $20 \%$ of the maximum of intensity. Lowest contour is three times the standard deviation of the noise, measured in regions external to the nebula

data set using the Shift-and-add method and the KnoxThompson method (Beckers et al. 1988, Eckart et al. 1990). The nebula was also observed by Rouan et al. (1993) in $K, L$ and $M$ bands and more recently in $H$ band by Roddier et al. (1995). These observations gave almost similar results and were obtained using adaptive optics technics.

It is interesting to notice that our $2.2 \mu \mathrm{m}$ reconstructed image (Fig. 2) is also very close to the $1.65 \mu \mathrm{m}$ Lucy deconvolution of the best image presented by Roddier et al. (1995). The X shape of the image already seen at $1.65 \mu \mathrm{m}$ is also present at $2.2 \mu \mathrm{m}$. These spikes can be interpreted in terms of radiative transfer of the stellar light in a biconus shaped dust shell producing the nebula. Yusef-Zadeh \& Morris (1984) have demonstrated that both the rectangular shape and the diagonal spikes of the Red Rectangle are the consequence of the scattering of light by the particles present in the envelope. These authors have performed a simulation of the radiative transfer with a density law for the envelope corresponding to a disk concentration of matter with a progressive decrease following the latitude angle $\theta\left(\theta=0^{\circ}\right.$ corresponds to the plane of the disk). A cut-off in the density law for $\theta \simeq 60^{\circ}$ may be responsible for the observed $\mathrm{X}$ shape of the nebula (Yusef-Zadeh \& Morris 1984).

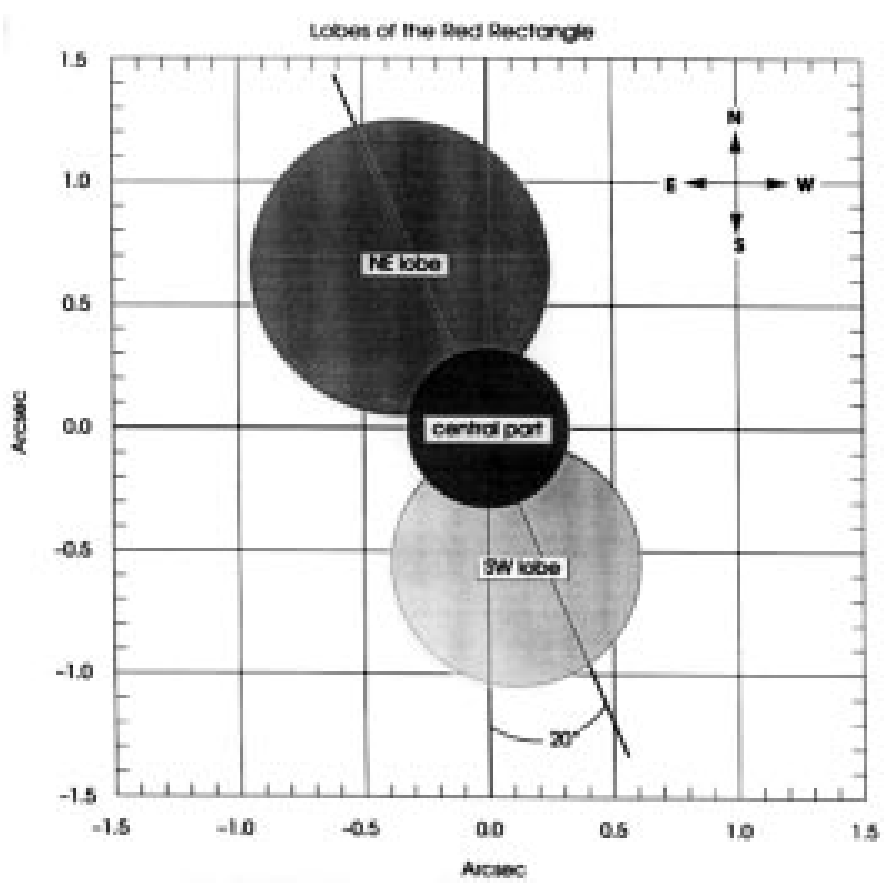

Fig. 5. Simplified geometry of the Red Rectangle based on the $M$-band reconstructed map. A central lobe $0.7^{\prime \prime}$ of diameter lies between 2 side lobes, $1.2^{\prime \prime}$ of diameter for the North-Eastern lobe and $1^{\prime \prime}$ for the South-Western one. The position angle (PA) defined by the centers of the side lobes is about $20^{\circ}$ (from North to East)

The rectangular shape is a known characteristics of the Red Rectangle in the red light of the visible. This rectangular shape holds at the first order up to $\lambda=5 \mu \mathrm{m}$. The scattering of light by dust is probably still efficient. Large particles of dust seem therefore to be present in the environment of HD 44179. This point was already established by Cohen et al. (1975) on the basis of polarimetric measurements.

\subsection{Red Rectangle central star subtraction}

To study the circumstellar environment, it is useful to separate the central stellar emission from the nebular flux (Hora et al. 1993). As instrumental PSF we used Gaussian functions of $0.2^{\prime \prime}$ in $K$ and $0.3^{\prime \prime}$ in $L^{\prime}$ and $M$ as described in the end of Sect. 4.9. The flux contribution from the central object HD $44179(V=9$, Cohen et al. 1975) can be removed from the nebular image by subtracting a scaled reference star image (Figs. 7a to 7c).

When the central star is scaled to subtract all the flux at the central position, a ring around the central position is left. In addition, these residual features are narrower than the instrumental PSF, so we regard them as being artificial. Instead we followed a different procedure to subtract the stellar component: assuming that it is more likely that there is a substantial contribution from a 


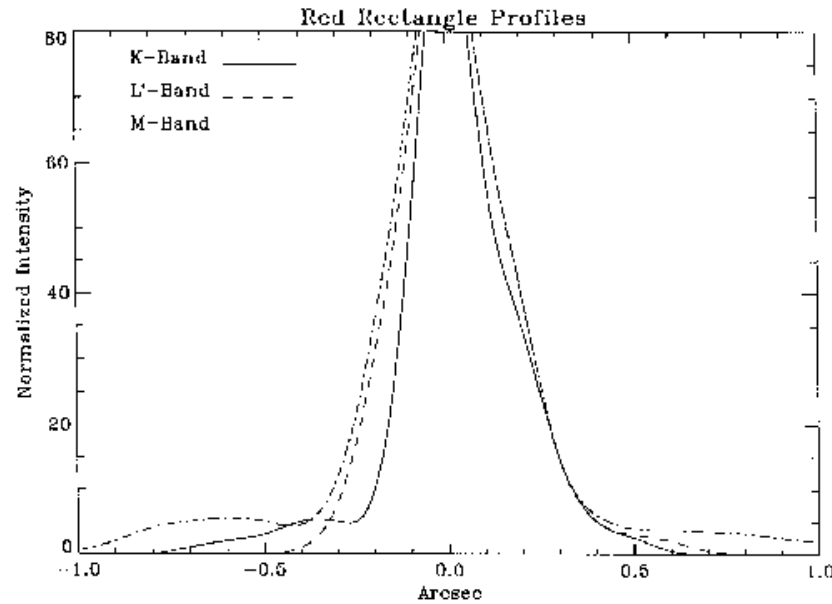

Fig. 6. Profiles of the Red Rectangle through the side lobes $\left(\mathrm{PA}=20^{\circ}\right)$. The profiles have been normalized so that the maximum level of intensity is 100 and the profiles are centered on the maximum of the central star. Each wavelength is assigned to a different line type, shown in the upper-left corner in the figure

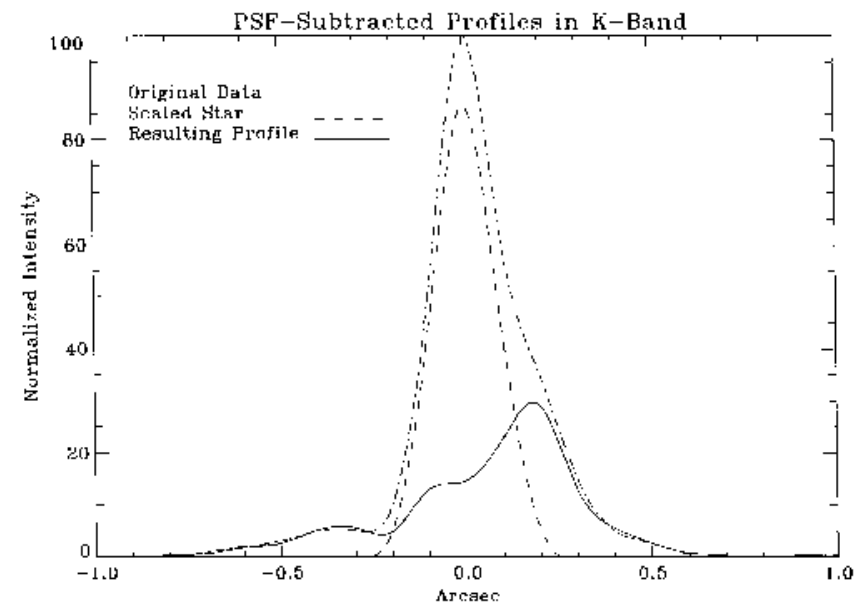

Fig. 7. a) PSF-subtracted profiles of the Red Rectangle at $K$. They show the original data (dot-dash line), the scaled standard star (dashed line), and the result when the star is subtracted (solid line)

spatially extended emission component in the central region (labeled "Central excess" in Table 1), we chose the unresolved central stellar flux (labeled "Star" in Table 1) to be the maximum possible value that does not create a central minimum.

Table 1 gives the near-IR relative fluxes of the Red Rectangle at each wavelength for each of the component of the image. Fluxes are measured in the central part and in the lobes defined in Fig. 5 (based on the $M$-band map). Errors are estimated via the standard deviation of the noise measured in regions external to the nebula.

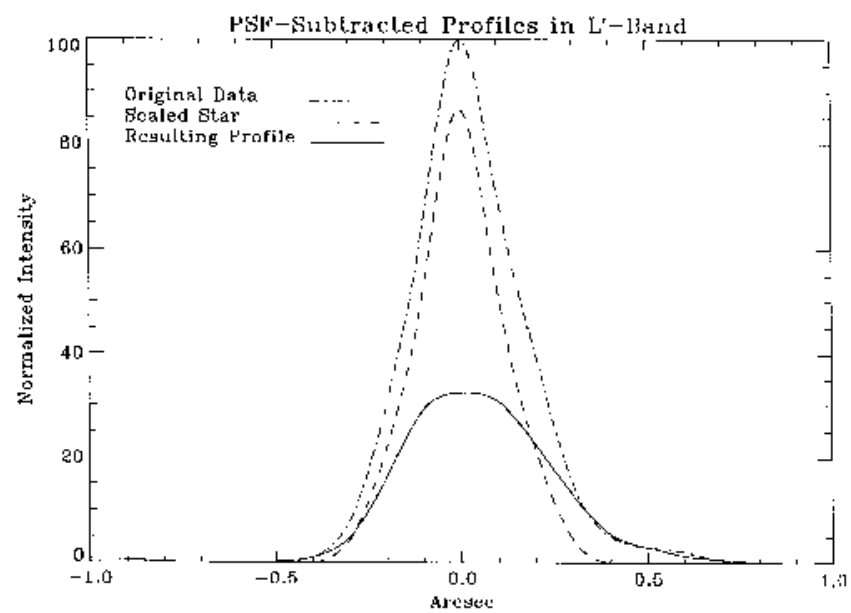

Fig. 7. b) PSF-subtracted profiles of the Red Rectangle at $L$ '. They show the original data (dot-dash line), the scaled standard star (dashed line), and the result when the star is subtracted (solid line)

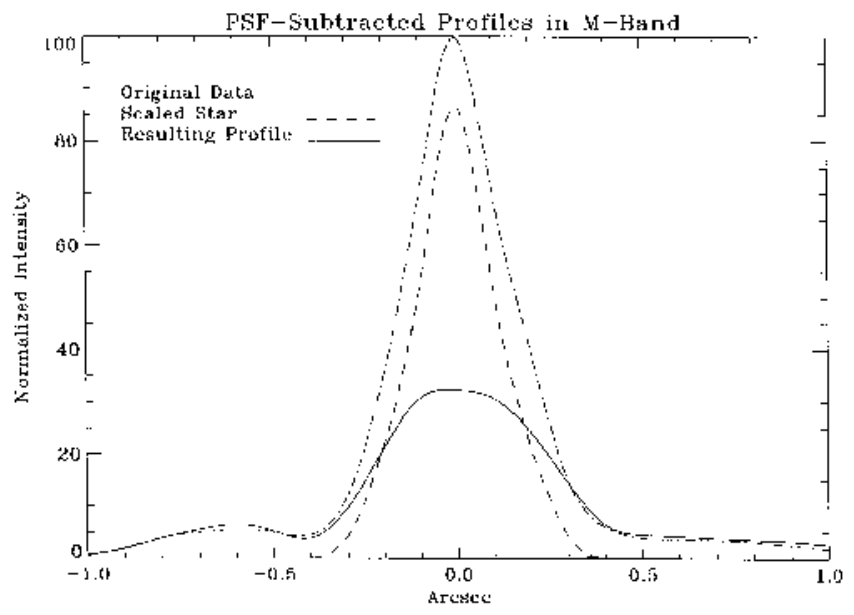

Fig. 7. c) PSF-subtracted profiles of the Red Rectangle at $M$. They show the original data (dot-dash line), the scaled standard star (dashed line), and the result when the star is subtracted (solid line)

\subsection{Broad-band spectral decomposition}

To determine the absolute infrared photometry in the $K, L$ and $M$ bands, the observations of Cohen et al. (1975), Allen et al. (1977), Kleinmann et al. (1978) and Gezari et al. (1987) summarized in Table 2 were used. The mean fluxes of the Red Rectangle in $K$, $L^{\prime}$ and $M$ bands, mainly based on Cohen et al. (1975) are estimated to be $F(K)=(1.55 \pm 0.06) 10^{-15}$ W.cm ${ }^{-2} \cdot \mu \mathrm{m}^{-1}([K]=3.5 \pm 0.1), F\left(L^{\prime}\right)=(2.45 \pm 0.1) 10^{-15}$ $\mathrm{W} \cdot \mathrm{cm}^{-2} \cdot \mu \mathrm{m}^{-1}\left(\left[L^{\prime}\right]=1.3 \pm 0.1\right)$ and $F(M)=(2.01 \pm 0.08)$ $10^{-15} \mathrm{~W} \cdot \mathrm{cm}^{-2} \cdot \mu \mathrm{m}^{-1}([M]=0.1 \pm 0.1)$.

Our spectral decomposition allows a new insight into the physics of the radiation mechanisms responsible for 
Table 1. Red Rectangle component fluxes (in \% of the total flux)

\begin{tabular}{cccc}
\hline \hline Filter & Star & Central excess & NE + SW lobes \\
\hline K $\ldots$. & $33 \pm 4$ & $25 \pm 3$ & $19 \pm 3$ \\
L. . . & $44 \pm 2$ & $35 \pm 3$ & $9 \pm 2$ \\
M . . & $28 \pm 3$ & $26+3$ & $20 \pm 4$ \\
\hline
\end{tabular}

the extended continuum emission of the Red Rectangle: Fig. 8 shows the decomposition of the $K, L^{\prime}$ and $M$ total flux into a central unresolved stellar component, a central extended excess and a combined side component (NE lobe $+\mathrm{SW}$ lobe) as we could obtained from this analysis.

The contribution of the nebula to the broadband spectrum apparently increases rapidly towards longer wavelengths. There are two ways out:

a) Scattering by big dust grains $(a \geq 0.5 \mu \mathrm{m})$. A problem with this solution is to determine the reality of this assumption and the abundance of the grains. According to Bregman (1977) from 8 to $13 \mu \mathrm{m}$ infrared spectroscopy measurements, amorphous carbon seems to be the most probable material responsible for the observed continuum. The polarimetric data of Cohen et al. (1975) suggests the presence of large particles.

b) Thermal emission by dust with a temperature of a few $100 \mathrm{~K}$. The problem here is to find out whether and at which distance from the star this warm dust exists. According to a radiative transfer model of Lopez et al. (1995), emission from dust heated by the radiation field of the star dominates in the near infrared.

More detailed broadband diffraction limited continuum imaging is required to distinguish between these possibilities. Most likely a combination of both is required to explain the properties of the extended emission.

\subsection{Clumps or binarity?}

Some of the features in our image are interesting to discuss in more details. The cut drawn through the center of the nebula (Fig. 6) shows in $K$ band a shoulder that appears more clearly after subtraction of the central component of the image (Fig. 7a). The shoulder peaks at about $0.2^{\prime \prime}$ to the North.

This characteristics was mentioned by Leinert \& Haas (1989). They detected in their $J, H$ and $K$ speckle measurements a non-compact feature roughly shifted of 0.15 arcsec to the North. Because the shoulder seems to be non-compact, it is more likely to be associated to some radiative transfer effect in the dust shell rather than to a companion star. The radiative transfer through a disk-like structure of dust may indeed produce a crescent shaped image of the envelope when the plane of the disk is tilted with respect to the line of sight of the observer (Lefèvre et al. 1982; Lopez 1994).

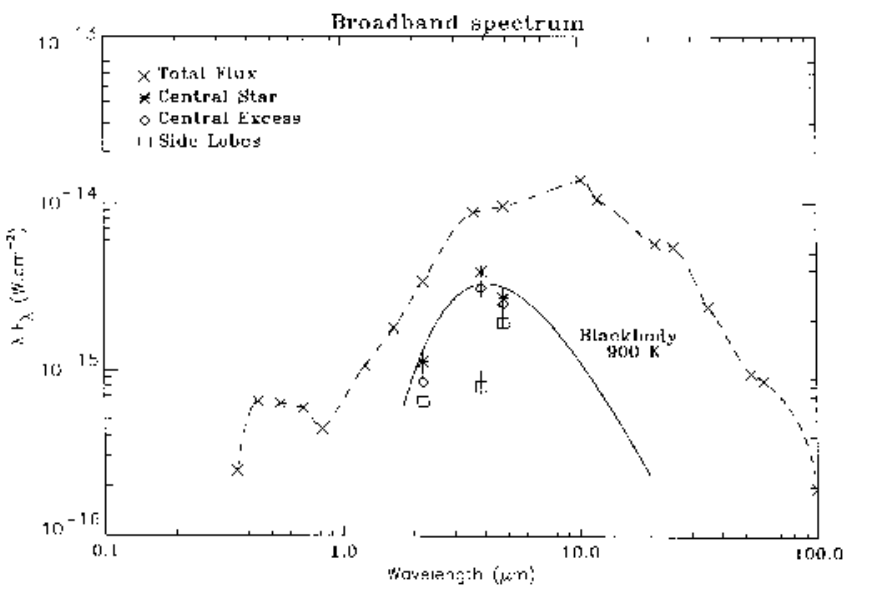

Fig. 8. Visible and infrared broadband spectrum of the Red Rectangle ( $X$ points, dotted line), based mainly on Cohen et al. (1975), Allen et al. (1977). Kleinmann et al. (1978) and IRAS measurements. Near-infrared fluxes of the reconstructed maps are shown at $K, L^{\prime}$ and $M$ for the central star (asterisks), the central excess (diamonds) and the side lobes (NE lobe $+\mathrm{SW}$ lobe, squares). Both axes are logarithmic. A blackbody curve (solid line) fitting the central part near-infrared measurements is shown for comparison

In the West and East directions, some components are present in our images, especially in the $K$-band $\left(0.75^{\prime \prime}\right.$ East, $0.4^{\prime \prime}$ West, $0.9^{\prime \prime}$ West and $1.2^{\prime \prime}$ West, relative to the center). Following Roddier et al. (1995) interpretation of their $1.65 \mu \mathrm{m}$ images, the edges of a slightly inclined dark disk could produce some of the observed bright structures in the East and West. HD 44179 is suspected to be a binary star (Cohen et al. 1975; Meadows et al. 1987). We may not exclude that one of the feature could be linked to the probably reddened companion star. Some clumps in the stellar environment could also be the origin of the detected structures.

In terms of stellar evolution it is of importance to measure the position of the stellar companion and to further estimate the orbital parameters of the system. Most postAGB sources present axisymmetrical images (Bujarrabal et al. 1992). Following the mechanism proposed by Morris et al. (1987), the mass loss of the late type star can be strongly perturbed by a second stellar component. The material is ejected in a non-spherical geometry and could even produce an accretion disk. We don't yet know at 
Table 2. Visible and infrared broad-band photometry of the Red Rectangle

\begin{tabular}{cccc}
\hline$\lambda(\mu \mathrm{m})$ & $\begin{array}{c}\text { Flux } \\
\left(10^{-16} \mathrm{~W}_{\mathrm{cm}}^{-2}\right)\end{array}$ & Bear & Ref. \\
\hline 0.36 & $2.46 \pm 0.03$ & $33^{\prime \prime}$ & 1 \\
0.438 & $6.47 \pm 0.05$ & $33^{\prime \prime}$ & 1 \\
0.5475 & $6.30 \pm 0.05$ & $33^{\prime \prime}$ & 1 \\
0.68 & $5.89 \pm 0.05$ & $33^{\prime \prime}$ & 1 \\
0.82 & $4.41 \pm 0.05$ & $33^{\prime \prime}$ & 1 \\
1.25 & $10.7 \pm 0.4$ & - & 2 \\
1.65 & $17.8 \div 0.7$ & $\ldots$ & 2 \\
2.2 & $34 \pm 1$ & $11^{4 *}$ & 1 \\
3.6 & $88 \pm 3$ & $11^{\prime \prime}$ & 1 \\
4.8 & $96 \pm 4$ & $22^{\prime \prime}$ & 1 \\
10.2 & $138 \pm 6$ & $22^{\prime \prime}$ & 1 \\
12 & $105.5 \pm 0.3$ & - & 4 \\
21 & $57 \pm 2$ & $11^{\prime \prime}$ & 1 \\
25 & $54.6 \pm 0.1$ & $\ldots$ & 4 \\
35 & $24 \pm 1$ & $22^{\prime \prime}$ & 3 \\
53 & $9.6 \pm 0.8$ & $22^{\prime \prime}$ & 3 \\
60 & $8.65 \pm 0.02$ & - & 4 \\
100 & $1.95 \pm 0.03$ & - & 4 \\
\hline
\end{tabular}

Ref.: (1) Cohen et al. 1975; (2) Allen et al. 1977; (3) Kleinmann et al. 1978; (4) IRAS Point Source Catalog (Gezari et al. 1987).

present if the bright star HD 44179 we observe is the mass loosing evolved star or its companion (Roddier et al. 1995).

\section{Conclusion}

We have shown that the bispectral analysis is a powerful method for reconstructing images observed in the near infrared in speckle mode. We have also shown the feasibility of two new numerical techniques included in the data processing. These are the iterative division (based on Van Cittert 1931) and a relaxation algorithm to calculate the complex source spectrum using measured triple products, calculated triple phases and the calculated power spectrum. The results obtained for the Red Rectangle are very encouraging. Images of the extended structure constituted by the dust shell of HD 44179 are in good agreement with those previously observed by means of adaptive optics. The physics of the near stellar environment of the source requires more high spatial resolution and multi-wavelength observations to be included in the interpretation. High spatial resolution performed at multiwavelength brings some useful information on the broadband spectrum of the object as it helps to separate the flux contribution from the nebula to the stellar radiation. With the angular resolution we presently have, some radiative transfer model could be performed to precise the charac- teristics of the outflow and disk surrounding HD 44179 and to distinguish between the contribution of scattered light from the central star and emission of warm dust.

Acknowledgements. We thank J.M. Beckers and S. Ridgway for having provided the KPNO $K$-band speckle data set. P.C. thanks MPG and CNRS for financial support of his stay at the MPE in Garching. J.-L. Starck, B. Sams, L.E. Tacconi-Garman, Y. Rabbia and A. Quirrenbach contributed with helpful discussions and comments.

\section{References}

Allen D.A., Hyland A.R., Longmore A.J., et al., 1977, ApJ 217, 108

Babcock H.W., 1953, PASP 65, 229

Bartelt H., Lohmann A., Wirnitzer B., 1984, Appl. Opt. 23, 3121

Beckers J.M., Christou J.C., Probst R.G., et al., 1988, First results with the NOAO 2-D speckle camera for infrared wavelengths, in: Proceedings of the ESO Conference on High-Resolution Imaging by Interferometry - Part 1. In: Merkle F. (ed.), European Southern Observatory, Garching bei München, Germany, p. 393

Bregman J.D., 1977, ApJ 89, 335

Bujarrabal V., Alcolea J., Planesas P., 1992, A\&A 257, 701

Cohen M., Anderson C.M., Cowley A., et al., 1975, ApJ 196, 179 
Cruzalèbes P., Schumacher G., Starck J.-L., 1992, J. Opt. Soc. Am. A 9, 708

Cunningham C.C., Anthony D., 1993, Icarus 102, 307

Drummond J., Eckart A., Hege E.K., 1988, Icarus 73, 1

Eckart A., Duhoux P.R.M., 1990, Infrared Speckle Reduction Software at the MPE, in: Proc. of a Conference on Astrophysics with Infrared Arrays, held at Tuscon, Arizona, February 19, 1990, Astron. Soc. Pac. Conf. Ser. In: Elston R. (eds.), p. 336

Ebersberger J., Weigelt G., 1986, A\&A 163, L5

Fried D.L., 1979, Opt. Acta 26, 597

Gatley I., 1987, BAAS 19, 1079

Gezari D.Y., Schmitz M., Mead J.M., 1987, Catalog of Infrared Observations, Appendix E. IRAS Point Source Catalog Data for CIO Sources. NASA Ref. Publ. 1196

Glindemann A., Lane R.G., Dainty J.C., 1992, Bispectral parameter estimation using least-square, in: Proceedings of the ESO Conference on High-Resolution Imaging by Interferometry II - Part 1. In: Beckers J.M. and Merkle F. (eds.), European Southern Observatory, Garching bei München, Germany, p. 243

Hardy J.W., Lefebvre J.E., Koliopoulos C.L., 1977, JOSA 67, 360

Hofmann K.-H., Weigelt G., 1986, A\&A 167, L15

Hora J.L., Deutsch L.K., Hoffmann W.F., Fazio G.G., Shivanandan K., 1993, ApJ 413, 304

Kleinmann S.G., Sargent D.G., Moseley H., et al., 1978, A\&A 65,139

Labeyrie A., 1970, A\&A 6, 85

Lacombe F., Tiphène D., Rouan D., Léna P., Combes M., 1989, A\&A 215, 211

Lefèvre J., Bergeat J., Daniel J.Y., 1982, A\&A 114, 341

Leinert Ch., Haas M., 1989, A\&A 221, 110

Lohmann A.W., Weigelt G., Wirnitzer B., 1983, Appl. Opt. 22,4028

Lopez B., 1994, Ph. D. Thesis, University of Nice - Sophia Antipolis
Lopez B., Mékarnia D., Lefèvre J., 1995, A\&A 296, 752

Lucy L.B., 1974, AJ 79, 745

Meadows P.J., Goods A.R., Wolstencroft R.D., 1987, MNRAS 225,43

Morris M., Guilloteau S., Lucas R., Omont A., 1987, ApJ 321, 888

Perrier C., 1988, NATO ASI Ser. C, Vol. 274, 99-111. In: Alloin D.M. and Mariotti J.-M. (eds.)

Roddier F., 1981, The effects of atmospheric turbulence in optical astronomy, in: Prog. Opt. XIX. In: Wolf E. (ed.), North Holland, p. 281

Roddier F., 1988, Phys. Rep. 170, 97

Roddier F., Roddier C., Graves J.E., Northcott M.J., 1995, ApJ 443, 249

Rouan D., 1993, Sub-arcsec IR imaging of transition objects, in: Proceeding of the ESO/CTIO Workshop on "Mass loss on the AGB and beyond", La Serena, Jan. 1992, Schwarz (ed.)

Tessier E., 1993, Ph. D. Thesis, University of Paris 6

Tessier E., Bouvier J., Lacombe F., 1994, A\&A 283, 827

Van Cittert P.H., 1931, Zeitschrift für Physics 69, 298

Von der Lühe O., Pehlemann E., 1988, Speckle masking imaging of extended sources, in: Proceedings of the ESO Conference on High-Resolution Imaging by Interferometry - Part 1. In: Merkle F. (ed.), European Southern Observatory, Garching bei München, Germany, p. 159

Weigelt G., 1977, Opt. Commun. 21, 55

Weitzel N., Haas M., Leinert C., 1992, Comparison of Knox-Thompson and bispectrum phase recovery for twodimensional near-infrared speckle data, in: Proceedings of the ESO Conference on High-Resolution Imaging by Interferometry II - Part 1. In: Beckers J.M. and Merkle F. (eds.), European Southern Observatory, Garching bei München, Germany, p. 511

Yusef-Zadeh F., Morris M., 1984, ApJ 278, 186 